\title{
Transcriptomics in Interferon- $\alpha$-Treated Patients Identifies Inflammation-, Neuroplasticity- and Oxidative Stress-Related Signatures as Predictors and Correlates of Depression
}

\author{
Nilay Hepgul', Annamaria Cattaneo',2, Kosh Agarwal', Sara Baraldi', Alessandra Borsini', Chiara Bufalino', \\ Daniel M Forton ${ }^{4}$, Valeria Mondelli', Naghmeh Nikkheslat', Nicola Lopizzo², Marco A Riva ${ }^{5}$, Alice Russell', \\ Matthew Hotopf' and Carmine M Pariante*,I \\ 'Department of Psychological Medicine, Institute of Psychiatry, Psychology and Neuroscience, King's College London, London, UK; ' IRCCS \\ Fatebenefratelli, University of Brescia, Brescia, Italy; ${ }^{3}$ Institute of Liver Studies, King's College Hospital, London, UK; ${ }^{4}$ Department of \\ Gastroenterology and Hepatology, St George's Hospital, London, UK; ${ }^{5}$ Department of Pharmacological and Biomolecular Sciences, University of \\ Milan, Milan, Italy
}

\begin{abstract}
Owing to the unique opportunity to assess individuals before and after they develop depression within a short timeframe, interferon- $\alpha$ $(\mathrm{IFN}-\alpha)$ treatment for chronic hepatitis $\mathrm{C}$ virus $(\mathrm{HCV})$ infection is an ideal model to identify molecular mechanisms relevant to major depression, especially in the context of enhanced inflammation. Fifty-eight patients were assessed prospectively, at baseline and monthly over 24 weeks of IFN- $\alpha$ treatment. New-onset cases of depression were determined using the Mini International Neuropsychiatric Interview (MINI). Whole-blood transcriptomic analyses were conducted to investigate the following: (I) baseline gene expression differences associated with future development of IFN- $\alpha$-induced depression, before IFN- $\alpha$, and (2) longitudinal gene expression changes from baseline to weeks 4 or 24 of IFN- $\alpha$ treatment, separately in those who did and did not develop depression. Transcriptomics data were analyzed using Partek Genomics Suite (1.4-fold, FDR adjusted $p \leqslant 0.05)$ and Ingenuity Pathway Analysis Software. Twenty patients (34\%) developed IFN- $\alpha$-induced depression. At baseline, 73 genes were differentially expressed in patients who later developed depression compared with those who did not. After 4 weeks of IFN- $\alpha$ treatment, 592 genes were modulated in the whole sample, representing primarily IFN- $\alpha$-responsive genes. Substantially more genes were modulated only in patients who developed depression ( $n=506$, compared with $n=70$ in patients who did not), with enrichment in inflammation-, neuroplasticity- and oxidative stress-related pathways. A similar picture was observed at week 24. Our data indicate that patients who develop IFN- $\alpha$-induced depression have an increased biological sensitivity to IFN- $\alpha$, as shown by larger gene expression changes, and specific signatures both as predictors and as correlates.

Neuropsychopharmacology (2016) 4I, 2502-25II; doi:I0.1038/npp.2016.50; published online 27 April 2016
\end{abstract}

\section{INTRODUCTION}

The development of clinically significant depression during interferon- $\alpha$ (IFN- $\alpha)$ therapy for chronic hepatitis $\mathrm{C}$ virus (HCV) infection is common, with an incidence of up to $45 \%$ (Asnis and De La Garza, 2006). Extensive research has been conducted to understand the biological systems involved in the development of IFN- $\alpha$-induced depression and to identify biological predictors associated with an enhanced risk to develop depressive symptoms. However, the molecular mechanisms are still unclear. Owing to the unique opportunity to assess individuals before and after they

* Correspondence: Professor CM Pariante, Department of Psychological Medicine, Institute of Psychiatry, Psychology and Neuroscience, King's College London, Cutcombe Road, London SE5 9RT, UK, Tel: + 020 7848 0807, E-mail: carmine.pariante@kcl.ac.uk

Received 14 January 2016; revised 7 March 2016; accepted 30 March 2016; accepted article preview online 12 April 2016 develop depression within a short period of time (weeks rather than months or years), this model can also crucially identify mechanisms relevant to major depression at large, or at least in the context of increased inflammation. Previous studies have used hypothesis-based approaches, focussed on specific biomarkers (Capuron et al, 2003; Lotrich et al, 2013; Raison et al, 2009). However, IFN- $\alpha$ activates many biological systems; therefore, a hypothesis-free approach may identify novel molecular mechanisms that predict or correlate with the development of depression.

Peripherally, IFN- $\alpha$ acutely induces the production and release of other innate immune cytokines such as interleukin-6 (IL-6) and tumor necrosis factor- $\alpha$ (TNF- $\alpha$ ) (Raison et al, 2008). These cytokines are putatively involved in the depressogenic action of IFN- $\alpha$. Higher serum or plasma levels of these (and other) pro-inflammatory markers have been shown to be associated with an increased risk of major depressive disorder (Dowlati et al, 2010). Our own work has shown increased inflammation in the blood of 
depressed patients when compared with healthy controls together with an association between higher cytokine levels and lack of antidepressant response (Cattaneo et al, 2013). Post-mortem gene expression studies show an upregulation of a variety of pro- and anti-inflammatory cytokines in the prefrontal cortex of depressed patients (Shelton et al, 2011), thus indicating that peripheral inflammation may correlate with central abnormalities. However, the link between increased peripheral inflammation and IFN- $\alpha$-induced depression is still unclear and studies report mixed results. For example, Wichers et al (2006) report a difference in plasma levels of IL-6 between patients who develop IFN- $\alpha$-induced depression and those who do not, but no difference in plasma levels of TNF- $\alpha$. Conversely, Raison et al (2008) report increased plasma concentrations of TNF- $\alpha$ to be significantly correlated with increased depression scores but no correlation was observed for IL-6.

A consolidated method to investigate the pathogenesis of psychiatric disorders is the use of peripheral blood to measure gene expression (mRNA) levels, which may be considered indicative of gene expression profiles in the brain (Hepgul et al, 2013). Several studies have shown that blood cells share $>80 \%$ of the transcriptome with other tissues, including the brain (Liew et al, 2006). A comparison of the transcriptional profiling of 79 human tissues showed that whole blood shares significant gene expression similarities with multiple brain tissues, in particular for genes encoding for neurotransmitter receptors and transporters, stress mediators, cytokines, hormones, and growth factors, all of which are relevant to depression (Sullivan et al, 2006). Global gene profiling in IFN- $\alpha$-treated cells has shown a number of IFN-stimulated genes (Wang and Campbell, 2005) such as IFN-induced $15-\mathrm{kDa}$ protein (ISG15), ubiquitin-specific proteinase 18 (USP18), IFN-induced $10-\mathrm{kDA}$ protein (IP-10 or chemokine (C-X-C motif) ligand 10 (CXCL10)), signal transducers and activators of transcription (STAT1), and IFN-induced guanylate-binding protein 3 (GBP3), and again with similarities between immune and brain cells (Wang et al, 2008). Of specific relevance to the present study, to date only five studies have investigated peripheral blood gene expression changes in the development of IFN- $\alpha$-induced depression. Three of these studies used a candidate gene approach, thus limiting the identification of novel or hitherto unknown mechanisms (Birerdinc et al, 2012; Krueger et al, 2011; Pawlowski et al, 2014), whereas two studies used transcriptomics (Felger et al, 2012; Schlaak et al, 2012). Felger et al (2012) used mRNA from isolated peripheral blood mononuclear cells (PBMCs) of $11 \mathrm{HCV}$ patients before and after 12 weeks of IFN- $\alpha$ treatment. They found 252 upregulated and 116 downregulated genes after 12 weeks of IFN- $\alpha$ treatment. However, the sample size was small (only four patients developed depressive symptoms) and only two genes were found to be differentially expressed in patients with depression: the 2'-5'-oligoadenylate synthetase 2 (OAS2; upregulated) and the high-affinity $\operatorname{IgE}$ receptor (FCER1A; downregulated). In the other transcriptomics study, blood mRNA was examined $12 \mathrm{~h}$ before and $12 \mathrm{~h}$ after the first injection of IFN- $\alpha$ (Schlaak et al, 2012). IFN- $\alpha$-induced depression (after at least 3 months of treatment) was found to be associated with upregulation of 15 genes, suggesting that very early responses to IFN- $\alpha$ are related to the development of depression.
In this study, we have used whole-blood transcriptomics to investigate, in a large sample of HCV patients, the following: (1) baseline gene expression differences associated with future development of IFN- $\alpha$-induced depression, before IFN- $\alpha$ administration, and (2) longitudinal changes in gene expression from baseline to treatment week 4 (TW4) and TW24 of IFN- $\alpha$, separately in those who did and did not develop depression. In addition, a small number of candidate cytokines were assessed in the plasma of patients at the same time points.

\section{MATERIALS AND METHODS}

\section{Study Design}

This was a prospective cohort study, evaluating patients at baseline and monthly over 24 weeks of IFN- $\alpha$ treatment. Blood samples for whole-blood mRNA analysis were collected in PAXgene Blood RNA Tubes (PreAnalytiX, Switzerland) and for plasma cytokine measurement in $2 \mathrm{ml}$ K3EDTA tubes (ThermoFisher Scientific, Massachusetts, USA) using standard protocols, at baseline and at TW4 and TW24. We recruited 58 participants from the outpatient liver departments of three London hospitals: King's College Hospital, Guy's and St Thomas' Hospital, and St George's Hospital. Eligible participants were adults with chronic HCV infection due to commence combination antiviral therapy with IFN- $\alpha$ and ribavirin for at least 24 weeks; this comprised weekly subcutaneous IFN- $\alpha$ injections $(1.5 \mu \mathrm{g}$ per $\mathrm{kg}$ of body weight) and daily ribavirin tablets (800-1400 mg/ day orally, in 2 divided doses). Exclusion criteria included the following: age below 18 years, current diagnosis (at baseline) of major depressive disorder, autoimmune disorder, current use of antidepressants, lack of English language, and co-infection with HIV or hepatitis B. Written informed consent was obtained from all participants. The research team did not interfere with the usual clinical practice; when the research team detected depression development during IFN- $\alpha$, this information was communicated to the treating hepatitis clinical team, who in turn decided whether or not to refer to liaison psychiatry services. The study was approved by the King's College Hospital Research Ethics Committee (Ref: 10/H0808/30).

\section{Questionnaires, Clinical Assessment, and Sample Description}

The Mini International Neuropsychiatric Interview (MINI) was administered at baseline, to assess current depression or a previous history of depression, and at follow-up assessments for the detection of new-onset cases of depression. The MINI is a structured diagnostic interview for psychiatric disorders according to the Diagnostic and Statistical Manual of Mental Disorders, 4th Edition and the International Statistical Classification of Diseases and Related Health Problems 10th Revision (Sheehan et al, 1998). For the purpose of this study, we only focused on the detection and diagnosis of major depressive episode. In addition, the severity of depressive symptoms was assessed using the Inventory of Depressive Symptomatology (IDS) (Rush et al, 1986). IDS scores were significantly higher at TW4 and TW24 when compared with baseline $(20.8 \pm 1.7$ vs $11.6 \pm 1.5$, $p<0.001$ and $22.9 \pm 2.0$ vs $11.6 \pm 1.5, p<0.001$, respectively), 
Table I Socio-Demographic and Clinical Characteristics of the Sample

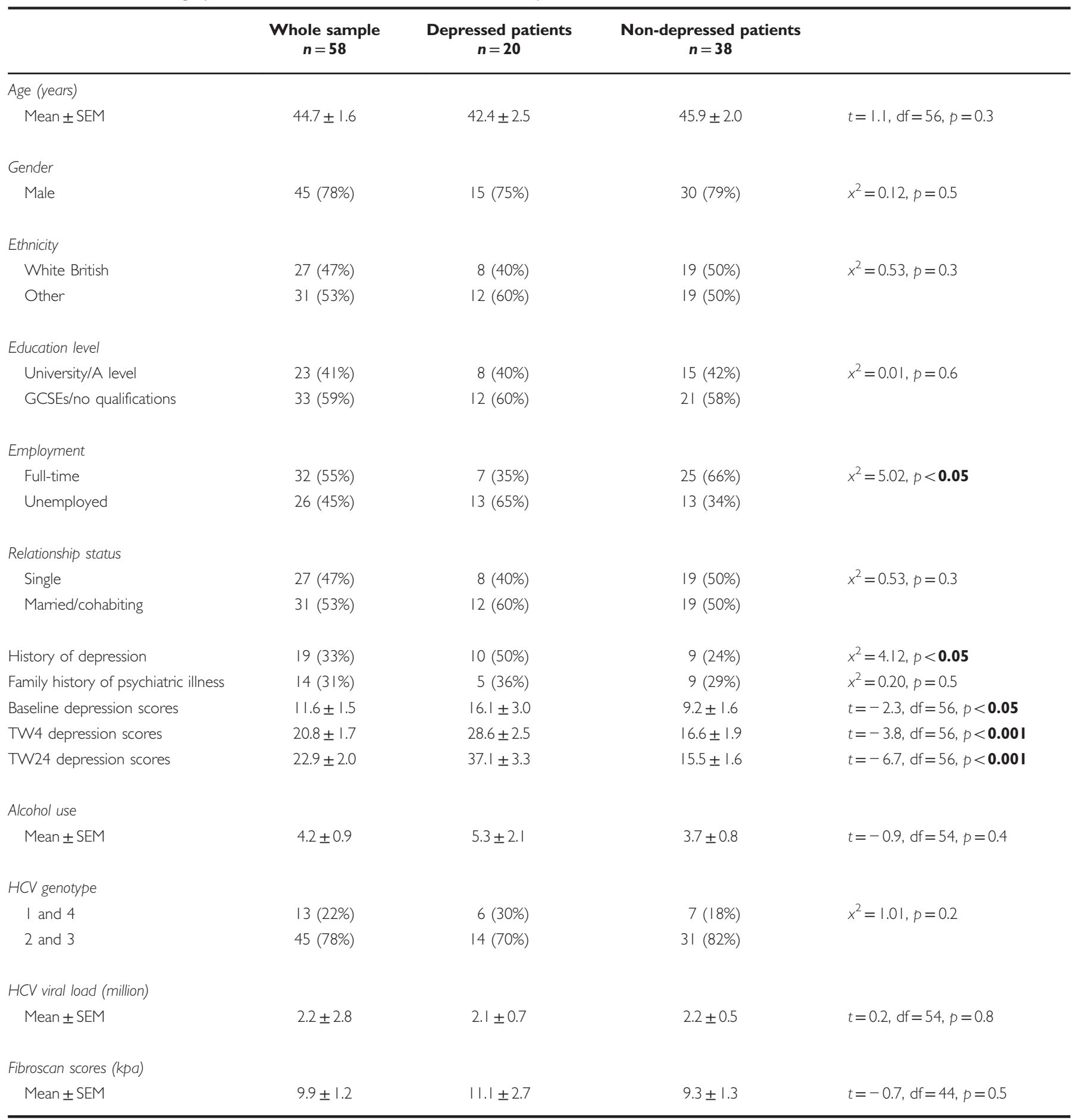

Numbers in bold indicate significant results.

whereas there was no significant difference between TW4 and TW24 (20.8 \pm 1.7 vs $22.9 \pm 2.0, p=0.2)$.

The depressed group was defined by a MINI diagnosis of major depressive episode at any time point during the 24 weeks. Four (7\%) patients developed depression by TW4 and 20 (34\%) patients developed depression by TW24, whereas 38 patients (66\%) did not develop depression. Two patients were started on antidepressant treatment after they developed IFN- $\alpha$-induced depression. The socio- demographic and clinical characteristics of the sample are presented in Table 1. Patients who developed depression were significantly more likely to have a previous history of depression, be unemployed, and have higher baseline depression scores. These three variables were interrelated and, indeed, those with a history of depression were more likely to be unemployed and to have higher depression scores at baseline (data not shown). Given this interrelation and the existing evidence for the presence of mood or anxiety 


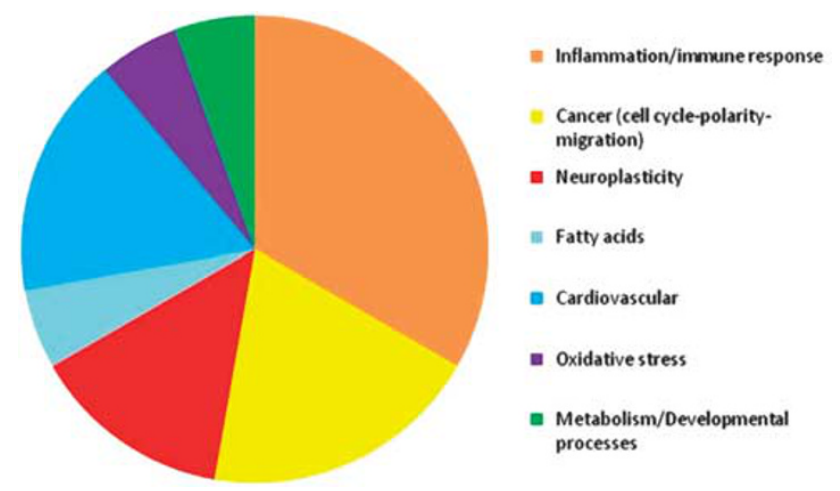

Figure I Role classification of pathways differentially modulated at baseline between patients who develop depression compared with those who do not.

symptoms before treatment as a risk factor (Dieperink et al, 2003; Lotrich et al, 2007), baseline depression scores were used as a covariate in all statistical analyses.

\section{RNA Isolation and Transcriptomics Analyses}

Isolation of total RNA was performed using the PAXgene blood miRNA kit according to the manufacturer's protocol (PreAnalytiX, Hombrechtikon, CHE). RNA quantity and quality were assessed by evaluation of the A260/280 and A260/230 ratios using a Nanodrop spectrophotometer (NanoDrop Technologies, Delaware, USA) and samples kept at $-80^{\circ} \mathrm{C}$ until processing for whole-genome transcriptomics analyses. Microarray assays were performed following the protocol described in the Affymetrix GeneChip Expression Analysis technical manual (Affymetrix, California, USA). Briefly, 250 ng RNA were used to synthesize cDNA with the Ambion WT Expression Kit (ThermoFisher Scientific), which was then purified, fragmented, labeled, and hybridized onto Human Gene 1.1 ST Array Strips (Affymetrix). The reactions of hybridization, fluidics, and imaging were performed on the Affymetrix GeneAtlas platform (Affymetrix) instrument according to the manufacturer's protocol. Validation of transcriptomics was performed using real-time PCR (full details in Supplementary Materials). Correlation between the Affymetrix and real-time values was 0.99 (data presented in Supplementary Table S1).

\section{Plasma Cytokine Measurement}

Blood samples were collected in $2 \mathrm{ml}$ K3EDTA tubes (ThermoFisher Scientific). On arrival to the laboratory, samples were centrifuged at $1500 \mathrm{~g}$ for $15 \mathrm{~min}$ at room temperature and plasma were removed and frozen at $-80^{\circ} \mathrm{C}$ until processing for cytokine measurement. All candidate proteins were measured using Magnetic Luminex Performance Multiplex Assay (R\&D Systems, Minneapolis, USA), using the customized 7-plex Human High Sensitivity Cytokine Premixed kit (R\&D, FCSTM14). From the cytokines available for 'high-sensitivity' measurement, we chose five cytokines known to be stimulated by IFN- $\alpha$ (IL- $1 \beta$, IL-2, IL-6, IFN- $\gamma$, and TNF- $\alpha$ (Taylor and Grossberg, 1998)) and two that are inhibited (IL-7 (Su et al, 1997) and IL-17A (Cui et al, 2014)). All samples were assayed according to the manufacturer's protocol and the results analyzed using SoftMax Pro V4.8 (full details in Supplementary Materials). IFN- $\gamma$ levels were below detection limit for most samples and therefore were not analyzed further.

\section{Statistical and Bioinformatic Analyses}

Data were analysed using IBM SPSS V20. Continuous variables are presented as mean \pm SEM. Differences in clinical and socio-demographic variables between patients who developed IFN- $\alpha$-induced depression and those who did not were analyzed using independent samples $t$-tests. Changes in depression scores between time points were analyzed using paired samples $t$-tests. A repeated-measures ANCOVA (with baseline IDS scores as a covariate) was performed, to test for differences in plasma cytokine levels as an effect of IFN- $\alpha$ treatment (time effect) and in relation to depression development (group effect). For gene expression data, CEL files were imported into Partek Genomics Suite V6.6 for data visualization and quality control. In summary (full details in Supplementary Materials), background correction was conducted using Robust Multi-strip Average (Irizarry et al, 2003) and Quantile Normalization (Bolstad et al, 2003) was used to normalize the distribution of probe intensities among different microarray strips. Summarization was conducted using a linear median polish algorithm (Tukey, 1977) to integrate probe intensities and compute expression levels for each gene transcript. To assess the effect of IFN- $\alpha$ treatment, a multiple linear contrast over time was performed and gene lists obtained by applying cutoffs of both $p$-value (FDR corrected) of $\leqslant 0.05$ and a minimum absolute fold change of 1.4. Ingenuity Pathway Analysis Software was used to identify regulation of molecular signaling pathways.

\section{RESULTS}

\section{Baseline Gene Expression Differences Predicting the Development of IFN- $\alpha$-Induced Depression}

We first examined differences in gene expression at baseline (before starting IFN- $\alpha$ ) and compared the profile of patients who subsequently did and who did not develop IFN- $\alpha$ induced depression. Using the above-mentioned cutoff criteria and baseline depression scores as a covariate, we found 73 differentially modulated genes (see Supplementary Table S2). Pathway analysis of these 73 genes identified 24 pathways including inflammation-, neuroplasticity- and oxidative stress-related pathways, such as IL-1 signaling, NRF2-mediated oxidative stress response, and axonal guidance signaling. These pathways are presented in Figure 1 and Table 2. In addition, we also compared plasma cytokine levels at baseline; however, there were no significant differences between the two groups (all $p$-values $>0.2$; see Supplementary Table S3).

\section{Genes Modulated by IFN- $\alpha$ at TW4 in the Whole Sample and in Relation to Development of IFN- $\alpha$-Induced Depression}

We identified genes modulated by IFN- $\alpha$, by comparing the expression profile of the sample at TW4 with the profile at baseline. IFN- $\alpha$ modulated 592 genes, including well-known 
Table 2 Pathways Differentially Modulated at Baseline Between Patients Who Develop Depression Compared With Those Who Do Not $(p \leqslant 0.05)$

\begin{tabular}{ll}
\hline Pathway & Molecules \\
\hline Ephrin B signaling & GNAT2, NCK2, ROCKI, GNG2 \\
Coagulation system & A2M, PROSI, SERPINDI \\
G alpha q signaling & RGSI8, PPP3RI, ROCKI, GNG2 \\
Ephrin receptor signaling & GNAT2, NCK2, ROCKI, GNG2 \\
NRF2-mediated oxidative stress response & DNAJC8, DNAJA2, GSTM3, GPX2 \\
Cardiac hypertrophy signaling & PPP3RI, GNAT2, ROCKI, GNG2 \\
CCR3 signaling in eosinophils & PLA2GI0, ROCKI, GNG2 \\
Netrin signaling & PPP3RI, NCK2 \\
Axonal guidance signaling & PPP3RI, GNAT2, NCK2, ROCKI, \\
CXCR4 signaling & GNG2 \\
Actin nucleation by ARP-WASP complex & NCK2, ROCKI \\
Role of NFAT in regulation of the immune & PPP3RI, GNAT2, GNG2 \\
response & \\
RhoGDI signaling & GNAT2, ROCKI, GNG2 \\
Thrombin signaling & GNAT2, ROCKI, GNG2 \\
Integrin signaling & NCK2, ROCKI, LIMSI \\
G Beta gamma signaling & GNAT2, GNG2 \\
Signalling by Rho family GTPases & GNAT2, ROCKI, GNG2 \\
IL-I signaling & GNAT2, GNG2 \\
Phospholipase C signaling & PPP3RI, PLA2GI0, GNG2 \\
fMLP signaling in neutrophils & PPP3RI, GNG2 \\
Androgen signalling & GNAT2, GNG2 \\
Extrinsic prothrombin activation pathway & PROSI \\
Glutathione redox reactions I & GPX2 \\
Relaxin signaling & GNAT2, GNG2 \\
\hline & \\
&
\end{tabular}

IFN- $\alpha$ targets such as IFN- $\alpha$-inducible protein 27 (IFI27, $\mathrm{FC}=+32.05), \quad$ IFN-induced protein 44-like (IFI44L, $\mathrm{FC}=+11.59), \quad$ USP18 $\quad(\mathrm{FC}=+4.39), \quad$ ISG15 ubiquitin-like modifier (ISG15, $\mathrm{FC}=+2.70)$, and CXCL10 $(\mathrm{FC}=+1.84)$. Subsequently, we investigated genes modulated by IFN- $\alpha$ separately in patients who developed depression $(n=20)$ and those who did not $(n=38)$. We found 506 genes modulated only in patients who developed depression (see Supplementary Table S4) and 70 genes modulated only in patients who did not develop depression (see Supplementary Table S5). We focussed our further analyses on the 506 genes specifically modulated in patients who developed depression. We found 224 upregulated and 284 downregulated genes, including genes previously found to be associated with depression such as CXCL10 $(\mathrm{FC}=+2.13)$ and insulin-like growth factor 2 mRNA binding protein 2 (IGF2BP2, $\mathrm{FC}=+1.88$ ). Pathway analysis of these 506 genes identified 65 pathways including those related to inflammation (IL-1, IL-6, and IL-8 signaling, glucocorticoid receptor (GR) signaling, triggering receptor expressed on myeloid cells 1 signaling, and nuclear factor- $\kappa \mathrm{B}$ cells (NF- $\kappa \mathrm{B}$ ) signaling), neuroplasticity (extracellular signal-regulated kinase 5 (ERK5) signaling and axonal guidance signaling), and oxidative stress (NRF2-mediated oxidative stress response, p53 signaling, and production of nitric oxide and reactive

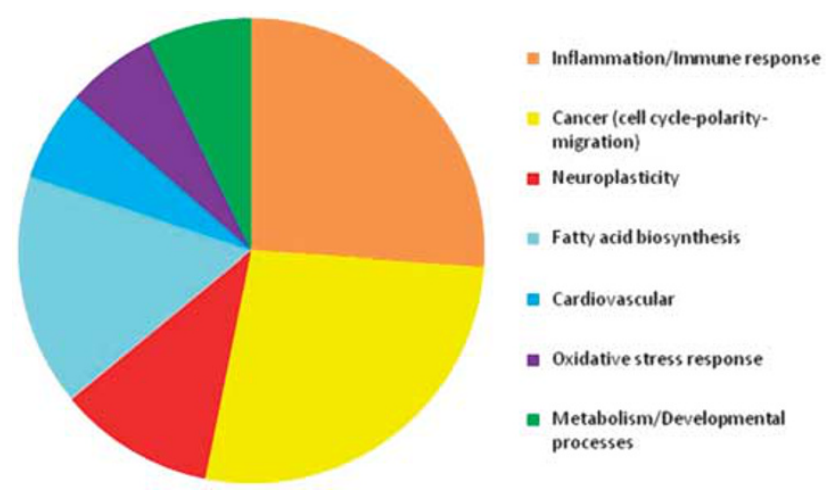

Figure 2 Role classification of pathways differentially modulated at treatment week 4 (TW4) specifically in patients who develop depression.

oxygen species in macrophages). These pathways are presented in Figure 2 and Table 3.

Finally, we conducted a two-way repeated-measure ANCOVA (with baseline depression scores as a covariate) to investigate changes in plasma cytokine levels between TW4 and baseline, and also in relation with depression development. We found a significant effect of IFN- $\alpha$ (time effect) for decreasing IL-1 $\beta(p=0.043)$ and increasing IL-6 $(p=0.013)$, IL-17A $(p=0.022)$, and TNF- $\alpha \quad(p<0.001)$, but no effect of depressive status (group effect, all $p$-values $>0.3$ ) nor time by group effect (all $p$-values $>0.2$ ) (see Supplementary Table S3).

\section{Genes Modulated by IFN- $\alpha$ at Week 24 in the Whole Sample and in Relation to Development of IFN- $\alpha$ - Induced Depression}

In order to assess whether IFN- $\alpha$-modulated changes persisted further down the treatment course, we compared the gene expression profile of patients at TW24 with the profile at baseline. Similar to TW4, modulation of several IFN- $\alpha$ target genes were observed in the whole sample, including IFI27 $(\mathrm{FC}=+34.20), \quad$ IFI $44 L \quad(\mathrm{FC}=+11.78), \quad$ USP $18 \quad(\mathrm{FC}=+4.72)$, ISG15 $(\mathrm{FC}=+2.80)$, and CXCL10 $(\mathrm{FC}=+2.52)$. In addition, we observed modulation of other known IFN- $\alpha$ target genes, which we did not see at TW4, such as GBP3 $(\mathrm{FC}=+1.45)$ and $S T A T 1,91 \mathrm{kDa}(\mathrm{FC}=+1.45)$. We found 285 genes modulated only in patients who developed depression (see Supplementary Table S6) and 121 genes modulated only in patients who did not develop depression (see Supplementary Table S7). We focussed our further analyses on the 285 genes that were modulated in patients who developed depression. These included 215 upregulated and 112 downregulated genes. Pathway analysis of these 285 genes identified 20 pathways. Interestingly, as indicated in Table 3, eight pathways were modulated at both TW4 and TW24, including ERK5 signaling, stress-activated protein kinase/Jun $\mathrm{N}$-terminal kinase (SAPK/JNK) signaling, and the glutathione redox reactions I pathway. The full list of 20 pathways and their role classifications can be seen in Supplementary Table S8 and Supplementary Figure S9, respectively.

Again, we conducted a two-way repeated-measure ANCOVA (with baseline depression scores as a covariate), to investigate changes in plasma cytokine levels between TW24 and baseline, and in relation with depression 
Table 3 Pathways Differentially Modulated at TW4 Specifically in Patients Who Develop Depression $(p \leqslant 0.05)$

Pathway Molecules

Aryl hydrocarbon receptor signaling

IL-6 signaling

ERK5 signaling ${ }^{\mathrm{a}}$

Pentose phosphate pathway ${ }^{\mathrm{a}}$

NRF2-mediated oxidative stress response

Phenylethylamine degradation $\mathrm{I}^{\mathrm{a}}$

NF- $\kappa B$ signaling

$L X R / R X R$ activation

Ephrin B signaling

Production of nitric oxide and reactive oxygen species in macrophages

IL-8 signaling

Phosphatidylglycerol biosynthesis II (non-plastidic)

GR signaling

Pentose phosphate pathway (non-oxidative branch)

Rapoport-Luebering glycolytic shunt

Glutathione redox reactions ${ }^{a}$

Fatty acid alpha oxidation

Pancreatic adenocarcinoma signaling ${ }^{a}$

RAR activation

Superpathway of inositol phosphate compounds

Pyrimidine deoxyribonucleotides de novo biosynthesis I

Role of macrophages, fibroblasts and endothelial cells in rheumatoid arthritis

$\mathrm{G}$ beta gamma signaling

Eicosanoid signaling

Colorectal cancer metastasis signaling

D-myo-inositol-5-phosphate metabolism

Granulocyte adhesion and diapedesis

Superpathway of D-myo-inositol (I,4,5)-trisphosphate metabolism

Prostanoid biosynthesis

PPAR signaling

EIF2 signaling ${ }^{\mathrm{a}}$

p53 signaling

Agranulocyte adhesion and diapedesis

Toll-like receptor signaling

Cholecystokinin/gastrin-mediated signaling

D-myo-inositol (I,4,5,6)-tetrakisphosphate biosynthesis

D-myo-inositol (3,4,5,6)-tetrakisphosphate biosynthesis

Cardiac hypertrophy signaling

Sorbitol degradation I

TREMI signaling

Pyrimidine ribonucleotides interconversion

Axonal guidance signaling

HGF signaling

Molecular mechanisms of cancer

Relaxin signaling

Pyrimidine ribonucleotides de novo biosynthesis

Acute phase response signaling
FOS, NCOR2, HSPBI, ILIB, ALDHIAI, NQO2, ALDH5AI, NFIA, MGSTI, TFDPI, MGST3, NRIPI

FOS, A2M, HSPBI, ILIRN, ILIB, TNFAIP6, CDI4, IL6R, AKT2, IL6ST

FOS, SGKI, GNAI2, WNKI, GABI, MAP3K3, IL6ST

TKT, PGD, TALDOI

FOS, DNAJA4, DNAJC8, DNAJC6, GCLC, ABCC4, DNAJA2, DNAJCI5, NQO2, MGSTI, MGST3

$\mathrm{ALDH} 2, \mathrm{AOC} 3$

BMPR2, TNFSFI3B, ILIRN, ILIB, AZI2, TLR6, PELII, AKT2, MAP3K3, PLCG2

NCOR2, SIOOA8, ILIRN, ILIB, MMP9, ORMI, CDI4, PTGS2

ACPI, EPHB4, GNAT2, GNAI2, ROCKI, GNG2

FOS, IFNGRI, NCF4, SI00A8, ORMI, NCFI, SIRPA, AKT2, MAP3K3, PLCG2

FOS, MMP9, CXCR2, PTGS2, CXCRI, GNAI2, ROCKI, BCL2LI, GNG2, AKT2

ABHD5, AGPAT9, PGSI

KAT2B, NCOR2, ILIB, PPP3CA, AKT2, FOS, A2M, ILIRN, DUSPI, SGKI, PTGS2, BCL2LI, NRIPI

TKT, TALDOI

BPGM, MINPPI

CLIC2, MGSTI, MGST3

ALDHIAI, PTGS2, ALDH2

NOTCHI, MMP9, PTGS2, E2F2, BCL2LI, TFDPI, AKT2

FOS, KAT2B, NCOR2, RPL7A, PML, DUSPI, ALDHIAI, AKT2, NRIPI, PRKARIA

PPPIR8, DUSPI, PPTC7, ACPI, PPP4RI, SIRPA, PPP3CA, MINPPI, INPP5A, PLCG2

AK5, NME4, RRM2B

TNFSFI3B, F2RLI, ILIB, IL6R, PPP3CA, AKT2, IL6ST, PLCG2, FOS, ILIRN, C5ARI, ROCKI, TLR6

GNAT2, GNA12, GNG2, AKT2, PLCG2, PRKARIA

CYSLTR2, DPEP2, PTGS2, FPR2, TBXASI

FOS, IFNGRI, MMP9, PTGS2, IL6R, BCL2LI, TLR6, GNG2, AKT2, IL6ST, PRKARIA

PPPIR8, DUSPI, PPTC7, ACPI, PPP4RI, SIRPA, PPP3CA, PLCG2

HSPBI, ILIRN, ILIB, MMP9, CXCLI0, C5ARI, CXCR2, FPR2, HRH2

IMPA2, MINPPI, INPP5A

PTGS2, TBXASI

FOS, NCOR2, ILIRN, ILIB, PTGS2, NRIPI

RPL5, RPL7A, RPLI3A, EIF4AI, RPL4I, RPS2, AKT2, RPSI5, AGO4

KAT2B, PML, BCL2LI, AKT2, RRM2B, PMAIPI

ILIRN, ILIB, MMP9, CXCLI0, C5ARI, CXCR2, CXCRI, AOC3, MYH9

FOS, ILIRN, ILIB, CDI4, TLR6

FOS, ILIRN, ILIB, PTGS2, GNAI2, ROCKI

PPPIR8, DUSPI, PPTC7, ACPI, PPP4RI, SIRPA, PPP3CA

PPPIR8, DUSPI, PPTC7, ACPI, PPP4RI, SIRPA, PPP3CA

HSPBI, GNAT2, GNAI2, IL6R, ROCKI, GNG2, PPP3CA, MAP3K3, PLCG2, PRKARIA

SORD

NLRPI2, ILIB, TLR6, AKT2, PLCG2

ENTPDI, AK5, NME4

MMP9, EPHB4, ADAM8, GNAT2, ARHGEFI2, GNAI2, TUBGI,PLXNCI, ADAMI9, PPP3CA, AKT2, PLCG2, PRKARIA, TUBAIA, ROCKI, GNG2

FOS, PTGS2, GABI, AKT2, MAP3K3, PLCG2

NOTCHI, GNAT2, ARHGEFI2, GNAI2, GABI, AKT2, PRKARIA, FOS, RALB, BMPR2, E2F2, BCL2LI, TFDPI, PMAIPI

FOS, MMP9, GNAT2, GNAI2, GNG2, AKT2, PRKARIA

ENTPDI, AK5, NME4

FOS, A2M, ILIRN,I LIB, ORMI, IL6R, AKT2, IL6ST 
Table 3 Continued

\begin{tabular}{|c|c|}
\hline Pathway & Molecules \\
\hline Role of NFAT in regulation of the immune response & FOS, SYK, GNAT2, GNAI2, GNG2, PPP3CA, AKT2, PLCG2 \\
\hline Xenobiotic metabolism signaling & NCOR2, GCLC, ILIB, ALDHIAI, UGT2B7, NQO2, ALDH5AI, MGSTI, MAP3K3, MGST3, NRIPI \\
\hline 3-Phosphoinositide degradation & PPPIR8, DUSPI, PPTC7, ACPI, PPP4RI, SIRPA, PPP3CA \\
\hline Triacylglycerol biosynthesis & ABHD5, AGPAT9, LPPR2 \\
\hline $\begin{array}{l}\text { Communication between innate and adaptive immune } \\
\text { cells }\end{array}$ & TNFSFI3B, ILIRN, ILIB, CXCLI0, TLR6 \\
\hline GM-CSF signaling & CSF2RA, BCL2LI, PPP3CA, AKT2 \\
\hline G alpha i signaling & RALB, CXCR2, FPR2, P2RYI4, GNG2, PRKARIA \\
\hline Aldosterone signaling in epithelial cells & HSPBI, DNAJC8, DNAJC6, DUSPI, SGKI, DNAJCI5, PLCG2 \\
\hline CDP-diacylglycerol biosynthesis I & ABHD5, AGPAT9 \\
\hline Histamine degradation & ALDHIAI, ALDH2 \\
\hline Salvage pathways of pyrimidine ribonucleotides & SGKI, AK5, CDK8, NME4, AKT2 \\
\hline Atherosclerosis signaling & SIO0A8, TNFRSFI4, ILIRN, ILIB, MMP9, ORMI \\
\hline Docosahexaenoic acid (DHA) signaling & ILIB, BCL2LI, AKT2 \\
\hline SAPK/JNK signaling ${ }^{\mathrm{a}}$ & MAP4K5, GNAI2, GNG2, GABI, MAP3K3 \\
\hline Aryl hydrocarbon receptor signaling & FOS, NCOR2, HSPBI, ILIB, ALDHIAI, NQO2, ALDH5AI, NFIA, MGSTI, TFDPI, MGST3, NRIPI \\
\hline
\end{tabular}

aPathways which were also modulated at TW24.

development. We found a significant effect of IFN- $\alpha$ (time effect) for increasing IL-6 ( $p=0.012)$, IL-17A $(p=0.008)$, and TNF- $\alpha(p<0.001)$, but again no effect of depressive status (group effect, all $p$-values $>0.4$ ) nor time by group interaction (all $p$-values $>0.1$ ) (see Supplementary Table S3).

\section{DISCUSSION}

To our knowledge, this is the first study to use a peripheral blood transcriptomics approach to identify both predictors of future development of IFN- $\alpha$-induced depression and biological pathways associated with the development of depression. Already before IFN- $\alpha$, patients who later develop depression show significant differences in the expression of several pathways compared with those who do not; in addition, by TW4 they show specific longitudinal changes in similar and additional pathways, related to inflammation, neuroplasticity, and oxidative stress. We replicate a number of previously identified IFN-responsive genes. For example, Wang et al (2008) listed ISG15, USP18, IP-10/CXCL10, STAT1, and IFN-induced GBP3 as some of the most highly expressed IFN responsive genes and we show changes in all of these genes following IFN- $\alpha$ administration. In addition, we replicate IFN- $\alpha$-stimulated genes found in cultured neurons including IRF7, PNPT1, and IFIH1 (Wang and Campbell, 2005). Finally, we replicate 100 genes (35\%) and 5 pathways, shown to be responsive to IFN- $\alpha$ by Felger et al (2012). These consistencies strengthen the validity of our findings.

Among the most significant differentially expressed genes at baseline (predictors), we find upregulation of ubiquitinfold modifier 1 (UFM1) and eukaryotic translation initiation factor $4 \mathrm{~B}$ (EIF4B) in patients who later develop
IFN- $\alpha$-induced depression. The exact functions of UFM1 are poorly understood; however, it has been shown to be involved in heart disease (Azfer et al, 2006) and diabetes (Lemaire et al, 2011) (conditions that present frequent comorbidity with depression), as well as schizophrenia (Rubio et al, 2013). EIF4B is a downstream component of the mammalian target of rapamycin (mTOR) signaling pathway. There is abundant evidence linking mTOR signaling to synaptic plasticity, memory, and psychiatric disorders (Hoeffer and Klann, 2010). Although we see increased expression of EIF4B in our patients who subsequently develop IFN- $\alpha$-induced depression, Jernigan et al (2011) report significant reductions in EIF4B (and mTOR) expression in the prefrontal cortex of depressed subjects. The sample used by Jernigan et al (2011) were on overage more than 10 years older and were predominantly suicide victims, which may account for these discrepant results. Also at baseline, we identified 24 pathways, some related to inflammation (IL-1 and CCR3 signaling), neuroplasticity (axonal guidance and netrin signaling), and oxidative stress (NRF2-mediated oxidative stress response and glutathione redox reactions I signaling). Some of these pathways were also significantly regulated longitudinally in patients who develop depression (see below).

Following 4 weeks of IFN- $\alpha$ treatment, we find that the number of modulated genes is more than seven times larger in patients who develop depression than in patients who do not, suggesting that patients who develop IFN- $\alpha$-induced depression have an increased biological sensitivity to IFN- $\alpha$. Of note, only four patients were depressed at TW4, while the majority became depressed between week 4 and $12(n=11)$ or between week 12 and $24(n=5)$; therefore, most patients classified as depressed in these analyses were on a trajectory to 
develop depression but had not yet developed it. As such, gene expression changes at TW4 could be conceptualized as early biological changes associated with future depression development, rather than as a consequence of a depressive status. In this regard, our findings are consistent with those of Capuron et al (2003) and Schlaak et al (2012), who have demonstrated that cortisol responses and, respectively, gene expression changes, after the first injection of IFN- $\alpha$, predict the development of depression after 12 weeks of treatment.

Our findings confirm and extend previous gene expression studies in patients with IFN- $\alpha$-induced depression. Felger et al (2012) used a different transcriptomics approach (isolated PBMCs and cross-sectional comparison at week 12). In relation to depression, they found two genes differentially regulated in those who develop depression: OAS2 (upregulated) and FCER1A (downregulated). We also found that these two genes were regulated in the same direction by IFN- $\alpha$ and found the change in OAS2 to be larger in patients who developed depression than in those who did not $(\mathrm{FC}=+4.13$ vs $\mathrm{FC}=+3.06$, respectively). Krueger et al (2011), Birerdinc et al (2012), and Pawlowski et al (2014) all used a candidate gene approach focused on key immune genes only and in smaller samples. They all found a variety of cytokines or cytokine target genes differentially modulated in depressed patients. Although we do not directly replicate any of these specific genes, we find an involvement of several inflammatory pathways including IL-6, IL-1, IL- 8 , and NF- $\kappa$ B. Finally, in their transcriptomics study, Schlaak et al (2012) identified 15 genes regulated $12 \mathrm{~h}$ after the first injection of IFN- $\alpha$ in 11 patients who developed depression (in comparison with 11 patients who did not develop depression). Although we do not replicate these genes exactly, we find five genes belonging to the same family of genes (guanylate-binding proteins, glutaredoxins, proteasome subunit- $\beta$ type, TNF superfamily members, and zinc finger proteins).

Our pathway analysis data show that patients who develop depression also exhibit modulation in oxidative stress pathways such as NRF2-mediated oxidative stress response, the glutathione redox reactions $\mathrm{I}$, and production of nitric oxide and reactive oxygen species in macrophages pathways. Glutathione has an important role in detoxifying various reactive oxygen species, which are known to induce oxidative stress (Franco et al, 2007). This finding is consistent with the evidence that depression is associated with increased oxidative and nitrosative stress, both in humans and in animal models (Bakunina et al, 2015). We also demonstrate the involvement of neuroplasticity pathways such as ERK5 signaling. Indeed, major depressive disorder may involve a reduced ability of neuronal systems to show adaptive plasticity, especially under stress conditions. There is a wealth of evidence, indicating reductions in various neurotrophic factors in the plasma and serum, and more recently also in the gene expression, of depressed patients (Cattaneo et al, 2010; Dwivedi et al, 2005; Otsuki et al, 2008). ERK5 is a member of the mitogen-activated protein kinase (MAPK) family that includes ERK1/2, p38, and JNK, and participates in the neuronal modulation of depression (Todorovic et al, 2009). Indeed, the modulation of the ERK5 signaling pathway is accompanied by alterations in the SAPK/JNK signaling pathway in our patients who develop IFN- $\alpha$-induced depression. Although ERK5 has not previously been implicated in the development of IFN- $\alpha$-induced depression, activation of p38 MAPK in peripheral blood lymphocytes following the initial injection of IFN- $\alpha$ has been shown to be implicated (Felger et al, 2011). One mechanism through which p38 MAPK can be depressogenic is by increasing the activity and expression of the serotonin transporter and by inhibiting GR function (Wang et al, 2004). This is in keeping with our finding for a modulation of the GR signaling pathway, specifically in our depressed group. Reduced GR function (glucocorticoid resistance) coupled with high levels of cortisol is an indicator of hypothalamic-pituitary-adrenal axis dysfunction and is one of the most replicated biological findings in depression, demonstrated also in gene expression studies (Matsubara et al, 2006). Glucocorticoid resistance has also been hypothesized to underlie the enhanced inflammation described in major depression (Pariante and Lightman, 2008).

We also measured a small number of candidate cytokines in the plasma of our patients. Although we find a significant effect of IFN- $\alpha$ treatment in the whole sample, we do not detect any differences between patients who developed IFN- $\alpha$-induced depression and those who did not. Indeed, previous studies examining peripheral inflammation and IFN- $\alpha$-induced depression have produced inconsistent results. For example, as mentioned previously, Wichers et al (2006) report a difference in plasma IL-6 levels between patients who develop IFN- $\alpha$-induced depression and those who do not, but no difference in plasma TNF- $\alpha$ levels. Conversely, Raison et al (2008) report increased plasma TNF- $\alpha$ concentrations to be significantly correlated with increased depression scores but no correlation was observed for IL-6. One study found no effect of IFN- $\alpha$ itself on plasma levels of key cytokines but instead found an increase in CSF concentrations (Raison et al, 2009). Although we found an effect of IFN- $\alpha$ on increasing IL-6, IL-17A, and TNF- $\alpha$, in general these studies and our findings confirm the notion that peripheral cytokines are not an accurate biomarker with reference to the effects of IFN- $\alpha$. As such, we believe investigating gene expression is a more accurate and reliable method, and indeed none of the previous gene expression studies conducted in IFN- $\alpha$-induced depression measured peripheral levels of cytokines.

Our findings are both biologically and clinically relevant. Studying such a clearly defined patient group at 'very high risk' of developing depression within a few weeks is a useful model to understand the pathogenesis of depression, especially depression in the context of enhanced inflammation, as it has been described after exposures to childhood trauma (Danese et al, 2008) or due to genetic predisposition (Bufalino et al, 2013). Of note, a recent transcriptomics study identified similar gene expression changes (in IL-6 and $\mathrm{NF}-\kappa \mathrm{B}$ signaling pathways) as correlates of antidepressant response to the TNF antagonist, infliximab, supporting the notion that our findings are relevant for this broader context (Mehta et al, 2013). There is also evidence that increased inflammation is associated with the lack of antidepressant response (Cattaneo et al, 2013; Guilloux et al, 2015), and therefore it is possible that the genes identified in our study are relevant to antidepressant response. Of note, in the context of $\mathrm{HCV}$ infection, biological predictors of IFN- $\alpha$ induced depression can still have a role in clinical practice, even in light of emerging IFN- $\alpha$-free treatment regimens. 
At present, there are several drugs licenced for use in $\mathrm{HCV}$ infection without IFN- $\alpha$ administration (Ryder, 2015); however, these treatments are not yet readily available for all viral genotypes and are highly expensive. As such, IFN- $\alpha$-induced depression remains a clinical burden for some populations.

There are some limitations of this study. First, we acknowledge there are tissue-specific differences in gene expression patterns and by using whole-blood mRNA we cannot understand which cell types are responsible for the changes we observe and how these may reflect changes in other tissues such as the brain. However, studies have shown a satisfactory degree of correlation between gene expression in the blood and in the brain (Cai et al, 2010; Liew et al, 2006; Sullivan et al, 2006), and indeed, as mentioned above, we replicate (in peripheral blood) the pattern of IFN-responsive genes previously described in IFN- $\alpha$-stimulated neurons (Wang and Campbell, 2005). Therefore, we believe that this issue does not detract from the impact our findings. Second, all of the patients received combination therapy with IFN- $\alpha$ and the antiviral agent ribavirin. Furthermore, although the use of antidepressants was an exclusion criterion at baseline, a very small number of depressed patients were prescribed antidepressants during IFN- $\alpha(n=2)$. As such, there may be some transcriptional and behavioral changes that could have been influenced by these pharmacological agents.

In conclusion, this study provides several lines of evidence for the possible molecular mechanisms involved in the impact of IFN- $\alpha$ on behavior. Beyond IFN- $\alpha$ treatment, the identified transcriptomics signatures could be used as biomarkers for the identification of individuals at risk of developing depression, especially in the context of high inflammation due to stress, physical illness, or genetic makeup, or to generate molecular targets for the discovery of new therapeutics in depression.

\section{FUNDING AND DISCLOSURE}

Professor Pariante and Dr Mondelli have received research funding from Johnson \& Johnson as part of a program of research on depression and inflammation. In addition, Professor Pariante and Dr Mondelli have received research funding from the Medical Research Council (UK) and the Wellcome Trust for research on depression and inflammation as part of two large consortia that also include Johnson \& Johnson, GSK, and Lundbeck. Dr Agarwal has received research funding from Bristol-Myers Squibb and Gilead, as well as consulting fees from Achillon, AbbVie, Astellas, Boehringer Ingelheim, Bristol-Myers Squibb, Gilead, Janssen, Merck, and Novartis. Dr Forton has received consulting fees from Merck, Boehringer Ingelheim, Roche, Janssen, AbbVie, BMS, and Gilead. Dr Hepgul, Dr Cattaneo, Dr Baraldi, Dr Bufalino, Ms Borsini, Professor Hotopf, Ms Russell, Mr Lopizzo, and Professor Riva all declare no conflict of interest.

\section{ACKNOWLEDGMENTS}

This work was supported by the grant 'Persistent Fatigue Induced by Interferon-alpha: A New Immunological Model for Chronic Fatigue Syndrome' (MR/J002739/1) and by the grant 'Immuno-psychiatry: A Consortium to test the Opportunity for Immunotherapeutics in Psychiatry' (MR/L014815/1), from the Medical Research Council (UK). Additional support has been offered by the National Institute for Health Research Mental Health Biomedical Research Centre in Mental Health at South London and Maudsley NHS Foundation Trust and King's College London. Dr Cattaneo and Professor Riva are also funded by the Eranet Neuron 'Inflame-D'.

\section{REFERENCES}

Asnis GM, De La Garza R 2nd (2006). Interferon-induced depression in chronic hepatitis $\mathrm{C}$ : a review of its prevalence, risk factors, biology, and treatment approaches. J Clin Gastroenterol 40: $322-335$.

Azfer A, Niu J, Rogers LM, Adamski FM, Kolattukudy PE (2006). Activation of endoplasmic reticulum stress response during the development of ischemic heart disease. Am J Physiol Heart Circ Physiol 291: H1411-H1420.

Bakunina N, Pariante CM, Zunszain PA (2015). Immune mechanisms linked to depression via oxidative stress and neuroprogression. Immunology 144: 365-373.

Birerdinc A, Afendy A, Stepanova M, Younossi I, Baranova A, Younossi ZM (2012). Gene expression profiles associated with depression in patients with chronic hepatitis $\mathrm{C}(\mathrm{CH}-\mathrm{C})$. Brain Behav 2: 525-531.

Bolstad BM, Irizarry RA, Astrand M, Speed TP (2003). A comparison of normalization methods for high density oligonucleotide array data based on variance and bias. Bioinformatics 19: 185-193.

Bufalino C, Hepgul N, Aguglia E, Pariante CM (2013). The role of immune genes in the association between depression and inflammation: a review of recent clinical studies. Brain Behav Immun 31: 31-47.

Cai CC, Langfelder P, Fuller TF, Oldham MC, Luo R, van den Berg LH et al (2010). Is human blood a good surrogate for brain tissue in transcriptional studies? BMC Genomics 11: 589.

Capuron L, Raison CL, Musselman DL, Lawson DH, Nemeroff CB, Miller AH (2003). Association of exaggerated HPA axis response to the initial injection of interferon-alpha with development of depression during interferon-alpha therapy. Am J Psychiatry 160: $1342-1345$.

Cattaneo A, Gennarelli M, Uher R, Breen G, Farmer A, Aitchison KJ et al (2013). Candidate genes expression profile associated with antidepressants response in the GENDEP study: differentiating between baseline 'predictors' and longitudinal 'targets'. Neuropsychopharmacology 38: 377-385.

Cattaneo A, Sesta A, Calabrese F, Nielsen G, Riva MA, Gennarelli M (2010). The expression of VGF is reduced in leukocytes of depressed patients and it is restored by effective antidepressant treatment. Neuropsychopharmacology 35: 1423-1428.

Cui F, Meng J, Luo P, Chen P (2014). IFN- alpha blocks IL-17 production by peripheral blood mononuclear cells in patients with chronic active hepatitis B Infection. BMC Infect Dis 14: 55.

Danese A, Moffitt TE, Pariante CM, Ambler A, Poulton R, Caspi A (2008). Elevated inflammation levels in depressed adults with a history of childhood maltreatment. Arch Gen Psychiatry 65: 409-415.

Dieperink E, Ho SB, Thuras P, Willenbring ML (2003). A prospective study of neuropsychiatric symptoms associated with interferon-alpha-2b and ribavirin therapy for patients with chronic hepatitis C. Psychosomatics 44: 104-112.

Dowlati Y, Herrmann N, Swardfager W, Liu H, Sham L, Reim EK et al (2010). A meta-analysis of cytokines in major depression. Biol Psychiatry 67: 446-457.

Dwivedi Y, Mondal AC, Rizavi HS, Conley RR (2005). Suicide brain is associated with decreased expression of neurotrophins. Biol Psychiatry 58: 315-324.

Felger JC, Alagbe O, Pace TW, Woolwine BJ, Hu F, Raison CL et al (2011). Early activation of p38 mitogen activated protein kinase is 
associated with interferon-alpha-induced depression and fatigue. Brain Behav Immun 25: 1094-1098.

Felger JC, Cole SW, Pace TW, Hu F, Woolwine BJ, Doho GH et al (2012). Molecular signatures of peripheral blood mononuclear cells during chronic interferon-alpha treatment: relationship with depression and fatigue. Psychol Med 42: 1591-1603.

Franco R, Schoneveld OJ, Pappa A, Panayiotidis MI (2007). The central role of glutathione in the pathophysiology of human diseases. Arch Physiol Biochem 113: 234-258.

Guilloux JP, Bassi S, Ding Y, Walsh C, Turecki G, Tseng G et al (2015). Testing the predictive value of peripheral gene expression for nonremission following citalopram treatment for major depression. Neuropsychopharmacology 40: 701-710.

Hepgul N, Cattaneo A, Zunszain PA, Pariante CM (2013). Depression pathogenesis and treatment: what can we learn from blood mRNA expression? BMC Med 11: 28.

Hoeffer CA, Klann E (2010). mTOR signaling: at the crossroads of plasticity, memory and disease. Trends Neurosci 33: 67-75.

Irizarry RA, Bolstad BM, Collin F, Cope LM, Hobbs B, Speed TP (2003). Summaries of Affymetrix GeneChip probe level data. Nucleic Acids Res 31: e15.

Jernigan CS, Goswami DB, Austin MC, Iyo AH, Chandran A, Stockmeier CA et al (2011). The mTOR signaling pathway in the prefrontal cortex is compromised in major depressive disorder. Prog Neuropsychopharmacol Biol Psychiatry 35: 1774-1779.

Krueger C, Hawkins K, Wong S, Enns MW, Minuk G, Rempel JD (2011). Persistent pro-inflammatory cytokines following the initiation of pegylated IFN therapy in hepatitis $\mathrm{C}$ infection is associated with treatment-induced depression. J Viral Hepat 18: e284-e291.

Lemaire K, Moura RF, Granvik M, Igoillo-Esteve M, Hohmeier HE, Hendrickx N et al (2011). Ubiquitin fold modifier 1 (UFM1) and its target UFBP1 protect pancreatic beta cells from ER stressinduced apoptosis. PLoS ONE 6: e18517.

Liew CC, Ma J, Tang HC, Zheng R, Dempsey AA (2006). The peripheral blood transcriptome dynamically reflects system wide biology: a potential diagnostic tool. J Lab Clin Med 147: 126-132.

Lotrich FE, Albusaysi S, Ferrell RE (2013). Brain-derived neurotrophic factor serum levels and genotype: association with depression during interferon-alpha treatment. Neuropsychopharmacology 38: 985-995.

Lotrich FE, Rabinovitz M, Gironda P, Pollock BG (2007). Depression following pegylated interferon-alpha: characteristics and vulnerability. J Psychosom Res 63: 131-135.

Matsubara T, Funato H, Kobayashi A, Nobumoto M, Watanabe Y (2006). Reduced glucocorticoid receptor alpha expression in mood disorder patients and first-degree relatives. Biol Psychiatry 59: 689-695.

Mehta D, Raison CL, Woolwine BJ, Haroon E, Binder EB, Miller $\mathrm{AH}$ et al (2013). Transcriptional signatures related to glucose and lipid metabolism predict treatment response to the tumor necrosis factor antagonist infliximab in patients with treatmentresistant depression. Brain Behav Immun 31: 205-215.

Otsuki K, Uchida S, Watanuki T, Wakabayashi Y, Fujimoto M, Matsubara T et al (2008). Altered expression of neurotrophic factors in patients with major depression. J Psychiatr Res 42: 1145-1153.

Pariante CM, Lightman SL (2008). The HPA axis in major depression: classical theories and new developments. Trends Neurosci 31: 464-468.

Pawlowski T, Radkowski M, Malyszczak K, Inglot M, Zalewska M, Jablonska J et al (2014). Depression and neuroticism in patients with chronic hepatitis $\mathrm{C}$ : correlation with peripheral blood mononuclear cells activation. J Clin Virol 60: 105-111.

Raison CL, Borisov AS, Majer M, Drake DF, Pagnoni G, Woolwine BJ et al (2009). Activation of central nervous system inflammatory pathways by interferon-alpha: relationship to monoamines and depression. Biol Psychiatry 65: 296-303.
Raison CL, Borisov AS, Woolwine BJ, Massung B, Vogt G, Miller AH (2008). Interferon-alpha effects on diurnal hypothalamicpituitary-adrenal axis activity: relationship with proinflammatory cytokines and behavior. Mol Psychiatry 15: 535-547.

Rubio MD, Wood K, Haroutunian V, Meador-Woodruff JH (2013). Dysfunction of the ubiquitin proteasome and ubiquitin-like systems in schizophrenia. Neuropsychopharmacology 38: 1910-1920.

Rush AJ, Giles DE, Schlesser MA, Fulton CL, Weissenburger J, Burns C (1986). The Inventory for Depressive Symptomatology (IDS): preliminary findings. Psychiatry Res 18: 65-87.

Ryder SD (2015). Chronic hepatitis C-what do the new drugs offer and who should get them first? Clin Med 15: 197-200.

Schlaak JF, Trippler M, Hoyo-Becerra C, Erim Y, Kis B, Wang B et al (2012). Selective hyper-responsiveness of the interferon system in major depressive disorders and depression induced by interferon therapy. PLoS ONE 7: e38668.

Sheehan DV, Lecrubier Y, Sheehan KH, Amorim P, Janavs J, Weiller E et al (1998). The Mini-International Neuropsychiatric Interview (M.I.N.I.): the development and validation of a structured diagnostic psychiatric interview for DSM-IV and ICD-10. J Clin Psychiatry 59(Suppl 20): 22-33.

Shelton RC, Claiborne J, Sidoryk-Wegrzynowicz M, Reddy R, Aschner M, Lewis DA et al (2011). Altered expression of genes involved in inflammation and apoptosis in frontal cortex in major depression. Mol Psychiatry 16: 751-762.

Su DM, Wang J, Lin Q, Cooper MD, Watanabe T (1997). Interferons alpha/beta inhibit IL-7-induced proliferation of CD4- CD8- CD3CD44+ CD25+ thymocytes, but do not inhibit that of CD4- CD8CD3- CD44- CD25- thymocytes. Immunology 90: 543-549.

Sullivan PF, Fan C, Perou CM (2006). Evaluating the comparability of gene expression in blood and brain. Am J Med Genet B Neuropsychiatr Genet 141B: 261-268.

Taylor JL, Grossberg SE (1998). The effects of interferon-alpha on the production and action of other cytokines. Semin Oncol 25(1 Suppl 1): 23-29.

Todorovic C, Sherrin T, Pitts M, Hippel C, Rayner M, Spiess J (2009). Suppression of the MEK/ERK signaling pathway reverses depression-like behaviors of CRF2-deficient mice. Neuropsychopharmacology 34: 1416-1426.

Tukey JW (1977). Exploratory Data Analysis. Addison-Wesley: Cambridge.

Wang J, Campbell IL (2005). Innate STAT1-dependent genomic response of neurons to the antiviral cytokine alpha interferon. J Virol 79: 8295-8302.

Wang J, Campbell IL, Zhang H (2008). Systemic interferon-alpha regulates interferon-stimulated genes in the central nervous system. Mol Psychiatry 13: 293-301.

Wang X, Wu H, Miller AH (2004). Interleukin 1alpha (IL-1alpha) induced activation of $\mathrm{p} 38$ mitogen-activated protein kinase inhibits glucocorticoid receptor function. Mol Psychiatry 9: 65-75.

Wichers MC, Kenis G, Leue C, Koek G, Robaeys G, Maes M (2006). Baseline immune activation as a risk factor for the onset of depression during interferon-alpha treatment. Biol Psychiatry 60: $77-79$.

This work is licensed under a Creative Commons Attribution 4.0 International License. The images
or other third party material in this article are included in the article's Creative Commons license, unless indicated otherwise in the credit line; if the material is not included under the Creative Commons license, users will need to obtain permission from the license holder to reproduce the material. To view a copy of this license, visit http://creativecommons.org/licenses/ by/4.0/ 